Article

\title{
An Improved Design for Chemomechanical Sensors: A Piezoresistive Pressure Sensor with a Mechanical Boss
}

\author{
Jeffrey Bates $^{1, *}$, Prashant Tathireddy ${ }^{2}$, Sebastian Buetefisch ${ }^{3}$ and Jules Magda ${ }^{4, *}$ \\ 1 Department of Materials Science and Engineering, University of Utah, 122 Central Campus Drive, \\ Room 304, Salt Lake City, UT 84112, USA \\ 2 Department of Electrical and Computer Engineering, University of Utah, 50 Central Campus Drive, \\ Room 3280, Salt Lake City, UT 84112, USA; E-Mail: p.tathireddy@utah.edu \\ 3 Department of Nanometrology, Physikalisch-Technische Bundesanstalt, Bundesallee 100, \\ Braunschweig 38116, Germany; E-Mail: sebastian.buetefisch@ptb.de \\ 4 Department of Chemical Engineering, University of Utah, 50 Central Campus Drive, Room 3290, \\ Salt Lake City, UT 84112, USA
}

* Authors to whom correspondence should be addressed; E-Mails: jeff.bates@utah.edu (J.B.); jj.magda@m.cc.utah.edu (J.M.); Tel.: +1-801-581-6915 (J.M.); Fax: +1-801-585-9291 (J.M.).

Received: 24 August 2013; in revised form: 14 September 2013 / Accepted: 16 October 2013 / Published: 28 October 2013

\begin{abstract}
Stimuli-responsive hydrogels can be used to convert miniature pressure sensors into novel chemomechanical sensors via confinement of the hydrogel sample between a porous membrane and a piezoresistive diaphragm. Chemomechanical sensors could prove beneficial in a variety of applications, including continuous monitoring of bioreactors and biomedical systems. In this study, one hydrogel composition with a high sensitivity to changes in $\mathrm{pH}$ was tested in two different chemomechanical sensors in order to compare the data obtained from each sensor design. In the first and older chemomechanical sensor design, a prefabricated hydrogel sample is loaded into the sensor chamber using a screw-on cap. In the newer sensor design, a thinner hydrogel is synthesized in situ and is held in place by a silicon boss that is mechanically connected to a piezoresistive diaphragm. The newer design results in a decreased chemomechanical sensor response time (by 60 times), and maintains a high sensitivity to changes in environmental stimuli.
\end{abstract}

Keywords: hydrogel based sensors; stimuli response; hydrogel thickness; microsensors; continuous analyte monitoring; $\mathrm{pH}$ response 


\section{Introduction}

Hydrogels are super absorbent network polymers consisting of three dimensional structures that can absorb and retain water and other aqueous fluids while maintaining the original structure. Hydrogels are made of water soluble monomer backbone molecules with a cross-linking molecule selected for either physical or chemical properties. Hydrogels are good candidates in biomedical applications because of their response to changes in the local environment. Hydrogels may swell or deswell depending on the conditions of the surrounding aqueous media. The swelling response is currently being harnessed in biological sensing applications for the detection of both analytes in solutions and biological compounds [1-8]. Hydrogels are known to respond to changes in $\mathrm{pH}$, glucose concentration, ionic strength, temperature, electric field, solvent composition and pressure. The swelling response of pH-responsive hydrogels occurs as nitrogen groups on the dimethylaminoethyl methacrylate (DMA) molecule become positively charged, which causes an electrostatic repulsion between neighboring DMA molecules.

In a chemomechanical sensor, this hydrogel swelling response is transduced into a measureable signal when the hydrogel exerts a stress on the diaphragm of a miniature pressure transducer. While chemomechanical sensors have been used with promising results in a number of different studies [9-24], improvements in design are still needed in order to improve sensor robustness and response kinetics without sacrifice of sensitivity.

There are several research groups who have worked on hydrogel-based sensors that measure the pressure exerted by a swelling response. The results of some of these projects are shown in Table 1.

Table 1. A comparison of $\mathrm{pH}-$ responsive hydrogel results from reference projects with the composition, hydrogel thickness and response time of each project.

\begin{tabular}{cccc}
\hline $\begin{array}{c}\text { Hydrogel Backbone/ } \\
\text { Sensing Group }\end{array}$ & $\begin{array}{c}\text { Hydrogel } \\
\text { Thickness }\end{array}$ & $\begin{array}{c}\text { pH Response } \\
\text { Time }\end{array}$ & Reference \\
\hline $\begin{array}{c}\text { Hydroxypropyl Methacrylate/ } \\
\text { Dimethylaminoethyl Methacrylate }\end{array}$ & $50 \mu \mathrm{m}$ & $15 \mathrm{~min}$ & $9-11$ \\
Polyvinyl Alcohol/Poly acrylic Acid & $50 \mu \mathrm{m}$ & $6 \mathrm{~min}$ & 14 \\
Acrylamide/Phenyl Boronic Acid & $400 \mu \mathrm{m}$ & & 16 \\
$\quad$ Hydroxypropyl Methacrylate/ & $400 \mu \mathrm{m}$ & $135 \mathrm{~min}$ & $17-20$ \\
Dimethylaminoethyl Methacrylate & $50 \mu \mathrm{m}$ & $30 \mathrm{~min}$ & 22 \\
$\begin{array}{c}\text { Polyvinyl Alcohol/Poly acrylic Acid } \\
\text { Polyvinyl Alcohol/Poly acrylic Acid }\end{array}$ & $40 \mu \mathrm{m}$ & $78 \mathrm{~min}$ & 23 \\
\hline
\end{tabular}

The data presented in Table 1 compares the hydrogel composition, thickness and response time across several research projects. Not all of the research projects provided results for the composition, thickness or response time in their projects because those parameters were not the main focus of their research. Furthermore, the focus of the research projects listed in Figure 1 is not the same as the research presented here. Some groups measure the swelling from dehydrated to a hydrated state [22], while others address the diffusion and swelling kinetics to characterize the swelling response [14]. These projects are provided here to illustrate the inconsistency of these parameters among research projects. One sensor assembly, referred to as the Han et. al. sensor [16], utilizes cylindrical samples of 
a hydrogel and a macro piezoresistive sensor to measure changes in the swelling pressure. Another group [23] created a sensor assembly with an elevated platform. This platform makes it possible to test hydrogel samples with a decreased thickness. This sensor utilizes microchannels for fluid exchange. The pedestal design was further investigated in this project, and is referred to as the piezoresistive pressure sensor with a mechanical Boss, or the Boss sensor.

Figure 1. The set up for the Han chemomechanical sensor includes a piezoresistive surface pressure sensing surface (A), a hydrogel sample of $400 \mu \mathrm{m}$ in thickness and $3.5 \mathrm{~mm}$ in diameter placed on the sensor $(\mathbf{B})$, a stainless steel sensor cap $(\mathbf{C})$ and a mesh membrane to allow fluid exchange (D).
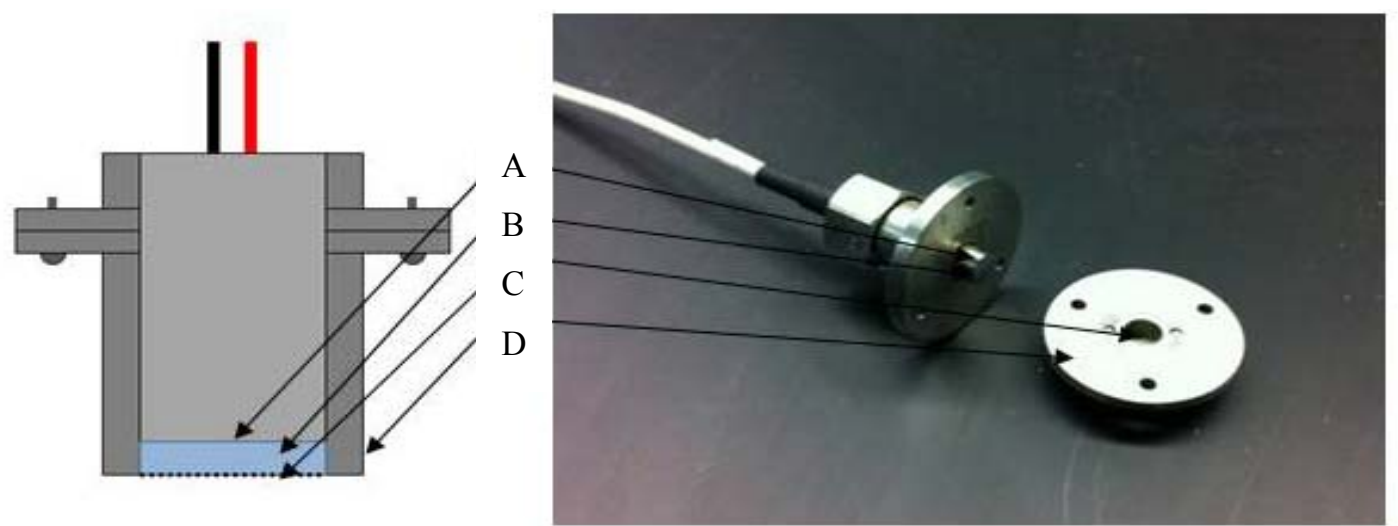

In this study, two different chemomechanical sensor designs are compared. In the first and older design, which dates back at least to 2002 [16], the hydrogel is synthesized in a mold and then placed into the sensor using a screw-on cap with a porous membrane top. This sensor design is referred to as the Han sensor [16]. This simple design has been successfully used in a number of studies [12-19], but is not well suited for use with thin hydrogels because of excessive compliance within the chamber that confines the hydrogel against the pressure transducer diaphragm [12]. In the second and newer design, the same type of hydrogel is synthesized in situ between a porous membrane and a boss, a protruding component on the sensor, which is mechanically connected to the pressure transducer diaphragm. This design is more difficult to fabricate but allows thinner hydrogels to be used, which should lead to smaller sensor response times. The objective of this study is to compare the response times and magnitudes of the two chemomechanical sensors using the same pH-responsive hydrogel, and to determine the advantages and disadvantages of each sensor.

\section{Experimental Methods}

\subsection{Materials}

The following monomers were used as received from Sigma Aldrich: 2-hydroxypropyl methacrylate (HPMA), dimethylaminoethyl methacrylate (DMA) and tetraethylene glycol dimethacrylate (TEGDMA). In addition, 2,2-dimethoxy-2-phenylacetophenone (DMPAP), a photoinitiator, and ethylene glycol (EG), a solvent for the pregel solution, were also obtained from Sigma Aldrich and used as received. Dulbecco's phosphate buffered saline (PBS) was mixed at 9.6 g/L 
in deionized water, and (3-aminopropyl) triethoxysilane (APTES) was prepared as 5\% in solution with ethanol. Both the PBS and APTES were obtained from Sigma Aldrich.

\subsection{Hydrogel Monolith Synthesis}

Hydrogel monoliths were synthesized in a mole ratio of 76.1 DMA, 2.2 HPMA, 0.6 TEGDMA and 21.1 EG. The pregel solution was purged with argon gas, and injected into a synthesis module creating a monolith with a thickness of $400 \mu \mathrm{m}$. The synthesis module was also purged with argon gas and consisted of two glass plates with a $400 \mu \mathrm{m}$ spacer. The injected pregel solution was exposed to ultraviolet light at $365 \mathrm{~nm}$ for $90 \mathrm{~s}$, which activated the photo initiator and resulted in free radical polymerization. After polymerization, the hydrogel monolith was removed from the synthesis module, washed with deionized water and stored in PBS for $24 \mathrm{~h}$ prior to conditioning. The hydrogel was conditioned by alternating ionic strength conditions every $4 \mathrm{~h}$ for at least 3 cycles. This allows the hydrogel to swell and deswell to remove unreacted monomers from the hydrogel matrix.

\subsection{Han et al. Chemomechanical Sensor Design Specifications}

The swelling pressure of the hydrogel samples was measured using two different chemomechanical sensors. The first, the Han sensor, consisted of a piezoresistive sensor (EPX Series, Measurement Specialties, Hampton, VA, USA) and a cap containing a porous mesh membrane (see Figure 1). This device encloses the piezoresistive sensor and the hydrogel, while allowing fluid exchange between the exposed surface of the hydrogel and the surrounding environment. The cylindrical sample of hydrogel (3.5 mm diameter and $400 \mu \mathrm{m}$ height) was placed in the chemomechanical sensor and held in place using the screw-on cap. The porous membrane was a stainless steel wire cloth mesh (120) from Small Parts, Inc., Logansport, IN, USA. The sensor was placed in a stirred temperature controlled bath, and the signal was transmitted to a PC using an Agilent 34970A Data Acquisition Device (Santa Clara, CA, USA).

\section{Converting the Signal}

The signal output of both sensors was collected in units of $\mathrm{mV}$. The sensitivity of the pressure sensor was characterized with a water column, and the signal was converted to units of Pascals with Equation (1).

$$
P=S V+X
$$

In this equation, $\mathrm{P}$ is the pressure calculated, $\mathrm{S}$ is the sensitivity of the sensor used, $\mathrm{V}$ is the voltage obtained from the pressure sensor and $\mathrm{X}$ is a scaling parameter based on the baseline data of the sensor obtained prior to sample testing.

\subsection{Boss Chemomechanical Sensor-Design Specifications}

A new chemomechanical sensor design was used with the miniaturized hydrogel. The sensor consisted of a silicon pressure diaphragm and a mesh cover. The surface of the pressure sensor was 
$1 \mathrm{~mm}^{2}$ with a distance between the boss and a porous mesh of $50 \mu \mathrm{m}$. Therefore, utilizing this pressure sensor limited the thickness of the hydrogel sample to $50 \mu \mathrm{m}$ (see Figure 2).

Figure 2. The image on the left shows the actual microsensor assembly. The image on the right shows a cross sectional illustration of the sensor assembly. In the image (A) is the mesh membrane, (B) is the hydrogel sample, (C) is the piezoresistive diaphragm, and $(\mathbf{D})$ is the boss.
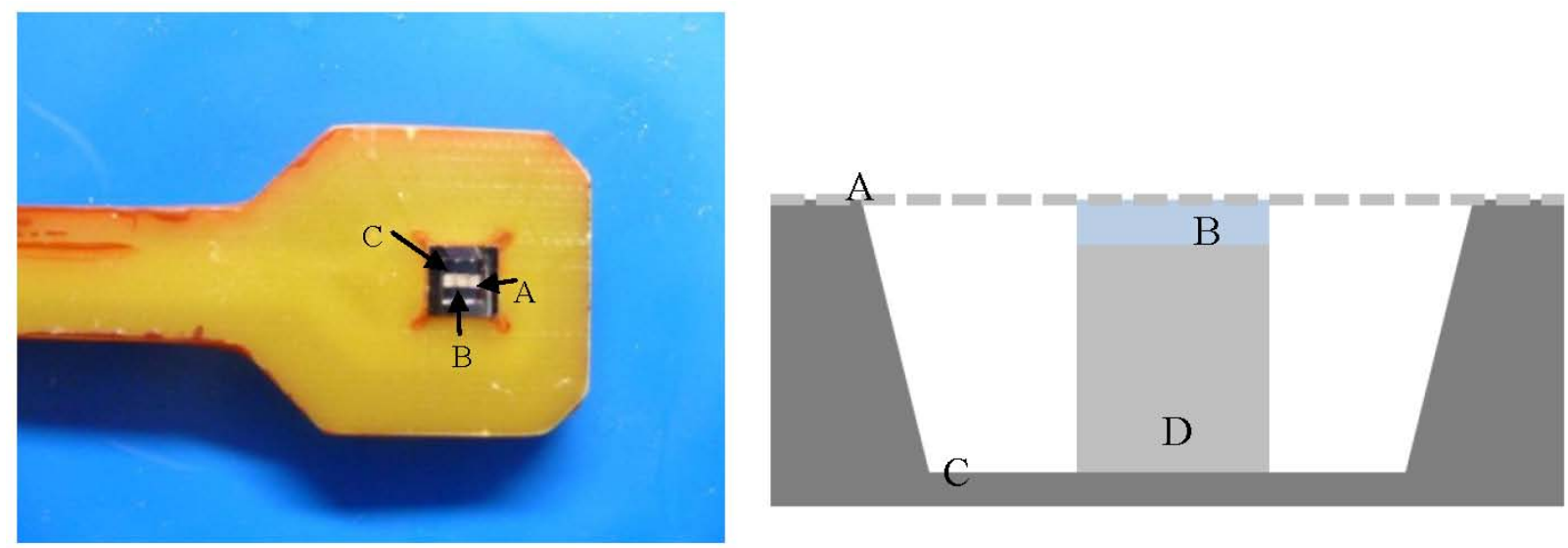

The swelling stress of the hydrogel is transmitted to the piezoresistive diaphragm via a silicon boss. The porous mesh was the same stainless steel wire used in the Han chemomechanical sensor design (see above). Data from the Boss sensor was also collected in units of voltage.

The response time for both sensors was determined as the duration of time from the initial change in the environmental conditions to the time where the hydrogel response came to equilibrium and maintained a stable response $(+/-0.1 \mathrm{mV})$.

\subsection{Surface Preparation of Boss Sensor}

The silicon surface of the boss was hydrophilic, therefore surface preparation with 5\% APTES in ethanol was used to increase adhesion of the hydrogel pregel solution to the silicon surface. A silicon wafer was used to simulate adhesion promotion and surface treatment prior to treating the sensor. One silicon wafer was treated with APTES and the other was controlled and left unchanged. The silicon wafer was treated with 5\% APTES by dipping the wafer in the solution five times and then immediately removing the residue in deionized water. The solvent was removed from the surface by low pressure argon blowing. A small amount of hydrogel pregel solution was synthesized with UV exposure on the surface of both treated and untreated silicon wafers, and adhesion was increased on the treated surface. After testing the experimental procedure on a silicon wafer, and determining that the adhesion was increased on the silicon surface, the surface of the pressure sensor was treated with APTES by following the same procedure. 


\subsection{In Situ Synthesis}

The pressure sensor was preassembled, and the hydrogel was injected through the mesh membrane. The hydrogel was synthesized in a glove box in an inert environment. The sensor was placed under a microscope and $1.5 \mu \mathrm{L}$ of the pregel solution was placed on the surface of the mesh membrane. The pregel solution was observed until it had passed through the mesh membrane. UV light with a wavelength of $365 \mathrm{~nm}$ was immediately exposed to the pregel solution until polymerization was complete. The hydrogel was not hydrated until testing began.

\subsection{Testing Procedures}

\subsubsection{Experiment 1: $\mathrm{pH}$ Response of $50 \mu \mathrm{m}$ Hydrogel Using Boss Sensor Design}

The Boss sensors were loaded with a hydrogel sample and tested from $\mathrm{pH} 4.0$ to 7.0 in $1 \mathrm{X}$ PBS at a constant ionic strength of $165 \mathrm{mM}$ and temperature of $25^{\circ} \mathrm{C}$ to determine the $\mathrm{pH}$ response time and magnitude. A cross sectional illustration of the microsensor is shown in Figure 2.

\subsubsection{Experiment 2: pH Response of $400 \mu \mathrm{m}$ Hydrogel Using Han Sensor Design}

A $3.5 \mathrm{~mm}$ diameter sample was removed from the hydrogel monolith using a biopsy tool and loaded into the Han sensor of Figure 1. The hydrogel was tested from pH 4.0 to 7.0 in $1 \mathrm{X}$ PBS at a fixed ionic strength of $165 \mathrm{mM}$ and at temperature of $25^{\circ} \mathrm{C}$ to determine the $\mathrm{pH}$ response time and magnitude for comparison to the Boss sensor.

\section{Results}

\subsection{Experiment 1: pH Response of $50 \mu \mathrm{m}$ Hydrogel in Boss Sensor Design}

A test was performed on the miniaturized hydrogel in the Boss design sensor (see Figure 3). The average swelling response time was $0.34 \mathrm{~h}$, with a magnitude of $2.04 \mathrm{kPa}$. The average deswelling response time was $0.07 \mathrm{~h}$, with a magnitude of $2.15 \mathrm{kPa}$.

Figure 3. Representative data showing 2 cycles of the $\mathrm{pH}$ response from the Boss sensor from $\mathrm{pH} 4.0$ to 7.0 .

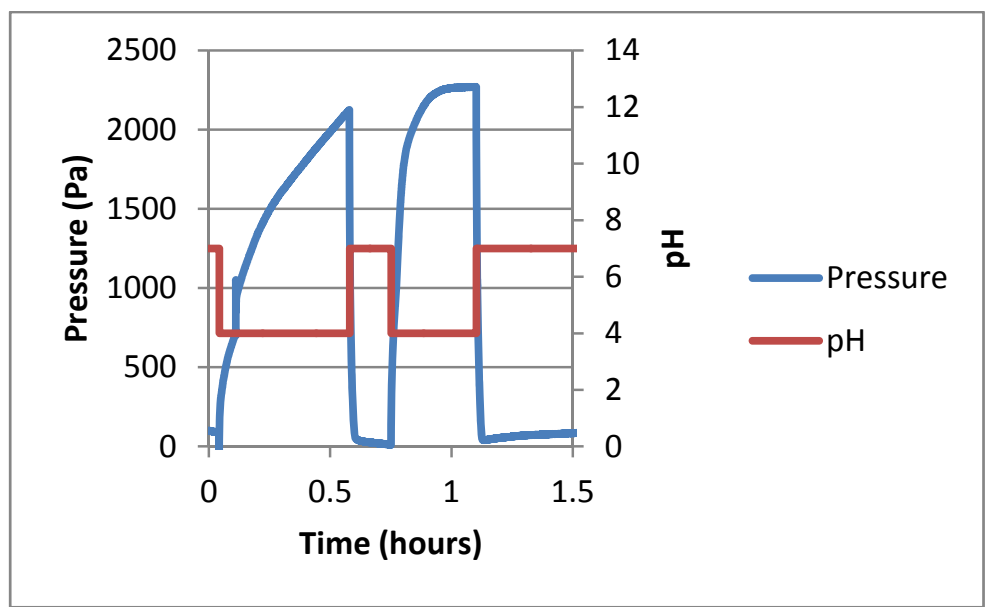




\subsection{Experiment 2: pH Response of $400 \mu m$ Hydrogel in Han Design Sensor}

A test was performed on a sample of the $400 \mu \mathrm{m}$ hydrogel sample in the Han sensor under the same $\mathrm{pH}$ change as the Boss sensor design (see Figure 4). The average swelling response time was $20 \mathrm{~h}$ with a magnitude of $1.63 \mathrm{kPa}$. The average deswelling response time was $30 \mathrm{~h}$, with a magnitude response of $1.22 \mathrm{kPa}$.

Figure 4. The representative $\mathrm{pH}$ response from $\mathrm{pH} 4.0$ to 7.0 of the $400 \mu \mathrm{m}$ hydrogel in the Han sensor.

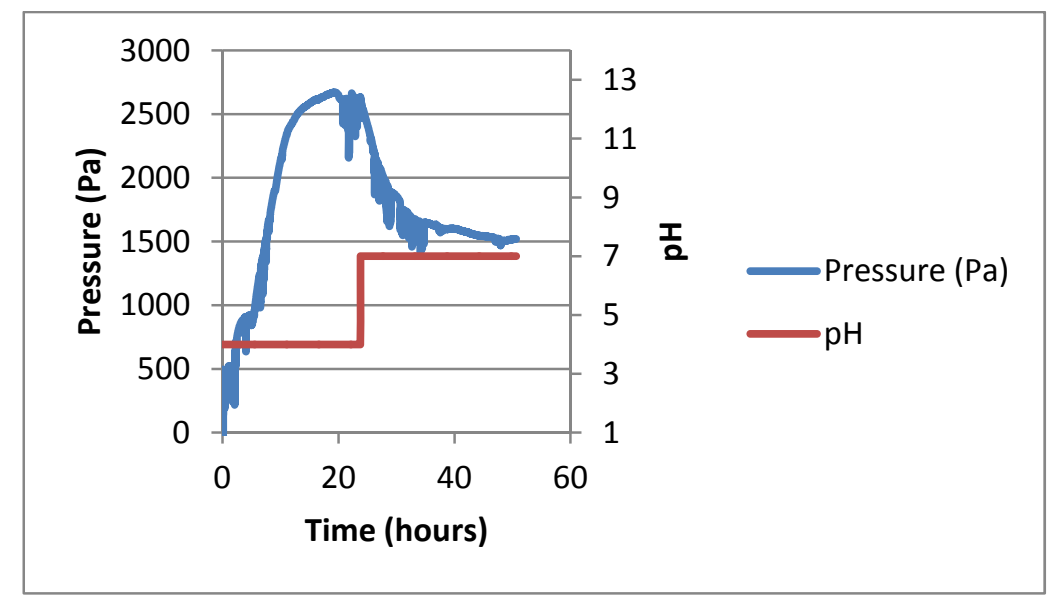

\section{Discussion}

The data given in Table 2 compare the response time and magnitude of the Boss sensor and the Han sensor for a $\mathrm{pH}$ change of 4.0 to 7.0. The $\mathrm{pH}$ response of the Boss sensor loaded with the $50 \mu \mathrm{m}$ hydrogel had a faster response time, $0.34 \mathrm{~h}$ compared to $20 \mathrm{~h}$ for the $400 \mu \mathrm{m}$ hydrogel. However, the response magnitude remained somewhat constant for the $50 \mu \mathrm{m}$ hydrogel, $2.04 \mathrm{kPa}$ compared to $1.63 \mathrm{kPa}$ for the $400 \mu \mathrm{m}$ hydrogel. Hence the new Boss sensor design successfully reduces the response time without sacrificing response magnitude. The hydrogel thickness reduction is the factor responsible for the reduced response time of the Boss sensor, which will likely have decreased the diffusion time through the hydrogel matrix. Assuming the sensor response is under diffusion control, the response time should be $8^{2}=64$ times smaller for the Boss sensor. The observed difference is 60 times smaller. The thickness of the hydrogel samples has an effect on the diffusion through the matrix. Assuming that the sensor response is under mass transfer control, the response time should depend on hydrogel thickness [24], which states that the hydrogel response is driven by diffusion. Therefore, if the diffusion is constant across the hydrogel, then the response time should depend on the second power of the hydrogel thickness.

Table 2. A comparison of response times and magnitudes of the experiments performed.

\begin{tabular}{ccc}
\hline Sensor Test & Response Time & Response Magnitude \\
\hline Boss $50 \mu \mathrm{m}$ & $0.34 \mathrm{~h}$ & $2.04 \mathrm{kPa}$ \\
Han $400 \mu \mathrm{m}$ & $20 \mathrm{~h}$ & $1.63 \mathrm{kPa}$ \\
\hline
\end{tabular}


The Han design provides advantages that include convenient reuse with different hydrogel samples, and the ability to use the sensor with hydrogel samples of uniform thickness. In addition, this design allows the researcher to load the sample with a pre-determined pressure value by adjustment of the screw-on cap prior to experimentation. On the other hand, the Boss sensor can be used to test thinner hydrogel samples, which results in a faster response time. In addition, the sensor can be easily miniaturized for use in biomedical monitoring.

\section{Conclusions}

Comparison has been made between two different designs of chemomechanical sensors: the Han design and the Boss design. The Han design is more convenient for reuse with different hydrogel samples. However, the Boss sensor can be used with thinner hydrogel samples to give faster sensor response kinetics without loss of sensitivity. When the Boss sensor is compared to the other sensors presented in Table 1, the Boss sensor has a simpler design that does not require the use of sampling of media solution, nor does it employ the use of microfluidic channels. Therefore the Boss sensor provides a useful application for continuous monitoring of biological systems.

\section{Conflict of Interest}

The authors declare no conflict of interest.

\section{References}

1. De, S.; Aluru, N.; Johnson, B.; Crone, W.; Beebe, D.; Moore, J. Equilibrium swelling and kinetics of pH-responsive hydrogels: Models, experiments and simulations. J. Microelectromechanical Syst. 2002, 11, 544-555.

2. Bienes, P.W.; Klosterkamp, I.; Menges, B.; Jonas, U.; Knoll, W. Responsive thin hydrogel layers from photo-cross-linkable poly ( $N$-isopropylacrylamide) terpolymers. Langmuir 2007, 23, 2231-2238.

3. Liu, M.; Guo, T. Preparation and swelling properties of crosslinked sodium polyacrylate. J. Appl. Polym. Sci. 2001, 82, 1515-1520.

4. Kuckling, D.; Hoffman, J.; Plotner, M.; Ferse, D.; Kretschmer, K.; Adler, H.; Arndt, K.; Reichelt, R. Photo cross-linkable poly( $N$-isopropylacrylamide) copolymers III: Micro-fabricated temperature responsive hydrogels. Polymer 2003, 44, 4455-4462.

5. Shin, J.; Braun, P.; Lee, W. Fast responsive photonic crystal $\mathrm{pH}$ sensor based on template photo-polymerized hydrogel inverse opal. Sens. Actuators B: Chem. 2010, 150, 183-190.

6. Iwata, T.; Suzuki, K.; Amaya, N.; Higuchi, H.; Masunaga, H.; Sasaki, S.; Kikuchi, H. Control of cross-linking polymerization kinetics and polymer aggregated structure in polymer-stabilized liquid crystalline blue phases. Macromolecules 2009, 42, 2002-2008.

7. Galeav, I.; Mattiason, B. Smart polymers and what they could do in biotechnology and medicine. Trends Biotechnol. 1999, 17, 335-340. 
8. Kurdikar, D.; Peppas, N. Method of determination of initiator efficiency: Application to UV polymerizations using 2,2-dimethoxy-2-phenylacetophenone. Macromolecules 1994, 27, 733-738.

9. Herber, S.; Olthius, W.; Bergveld, P.; van den Berg, A. Exploitation of a pH-sensitive hydrogel disk for $\mathrm{CO}_{2}$ detection. Sens. Actuators B: Chem. 2004, 103, 284-289.

10. Herber, S.; Bomer, J.; Olthius, W.; Bergveld, P.; van den Berg, A. A miniaturized carbon dioxide gas sensor based on sensing of $\mathrm{pH}$-sensitive hydrogel swelling with a pressure sensor. Biomed. Microdevices 2005, 7, 197-204.

11. Ter Steege, R.; Herber, S.; Olthius, W.; Bergveld, P.; van den Berg, A.; Kolkman, J. Assessment of a new prototype hydrogel $\mathrm{CO}_{2}$ sensor; comparison with air tonometry. J. Clin. Monit. Comput. 2007, 21, 83-90.

12. Lin, G.; Chang, S.; Kuo, C.; Magda, J.; Solzbacher, F. Free swelling and confined smart hydrogels for applications in chemomechanical sensors for physiological monitoring. Sens. Actuators B: Chem. 2009, 136, 186-195.

13. Schulz, V.; Guenther, M.; Gerlach, G.; Magda, J.; Tathireddy, P.; Rieth, L.; Solzbacher, F. In-vitro investigations of a $\mathrm{pH}$ - and ionic-strength-responsive polyelectrolyte hydrogel using a piezoresistive microsensor. Smart Struct. Mater. Nondestruct. Eval. Health Monitor. Diagn. 2009, $7827,1-16$.

14. Gerlach, G.; Guenther, M.; Sorber, J.; Suchanek, G. Chemical and pH sensors based on the swelling behavior of hydrogels. Sens. Actuators B: Chem. 2005, 111, 555-561.

15. Horkay, F.; Tasaki, I.; Basser, P.J. Osmotic swelling of polyacrylate hydrogels in physiological salt solutions. Biomacromolecules 2000, 1, 84-90.

16. Han, I.S.; Han, M.; Kim, J.; Lew, S.; Lee, Y.J.; Horkay, F.; Magda, J.J. Constant-volume hydrogel osmometer: A new device concept for miniature biosensors. Biomacromolecules 2002 , $3,1271-1275$.

17. Orthner, M.P.; Buetefisch, S.; Magda, J.; Rieth, L.W.; Solzbacher, F. Development, fabrication, and characterization of hydrogel based piezoresistive pressure sensors with perforated diaphragms. Sens. Actuators A: Phys. 2010, 161, 29-38.

18. Avula, M.; Busche, N.; Cho, S.H.; Tathireddy, P.; Rieth, L.W.; Magda, J.J.; Solzbacher, F. Effect of Temperature Changes on the Performance of Ionic Strength Biosensors Based on Hydrogels and Pressure Sensors. In Proceedings of the 33rd Annual International Conference of the IEEE EMBS, Boston, MA, USA, 3 August 2011; pp. 1855-1858.

19. Lin, G.; Chang, S.; Hao, H.; Tathireddy, P.; Orthner, M.; Magda, J.; Solzbacher, F. Osmotic swelling pressure response of smart hydrogels suitable for chronically implantable glucose sensors. Sens. Actuators B: Chem. 2010, 144, 332-336.

20. Tathireddy, P.; Avula, M.; Lin, G.; Cho, S.H.; Guenther, M.; Schulz, V.; Gerlach, G.; Magda, J.J.; Solzbacher, F. Smart Hydrogel Based Microsensing Platform for Continuous Glucose Monitoring. In Proceedings of the 32nd Annual International Conference of the IEEE EMBS, Buenos Aires, Argentina, 4 August 2010; pp. 677-679.

21. Lei, M.; Baldi, A.; Nuxoll, E.; Siegel, R.A.; Ziaie, B. A hydrogel-based implantable micromachined transponder for wireless glucose measurement. Diab. Technol. Therap. 2006, 8, $112-122$. 
22. Gerlach, G.; Guenther, M.; Suchaneck, G.; Sorber, J.; Arnt, K.; Richter, A. Application of sensitive hydrogels in chemical and pH sensors. Macromol. Symp. 2004, 210, 403-410.

23. Trinh, Q.T.; Gerlach, G.; Sorber, J.; Arndt, K. Hydrogel-based piezoresistive pH sensors, design, simulation and output characteristics. Sens. Actuators B: Chem. 2006, 117, 17-26.

24. Tanaka, T.; Fillmore, D.J. The kinetics of swelling of gels. J. Chem. Phys. 1979, 70, 1214-1218.

(C) 2013 by the authors; licensee MDPI, Basel, Switzerland. This article is an open access article distributed under the terms and conditions of the Creative Commons Attribution license (http://creativecommons.org/licenses/by/3.0/). 